\title{
Predictive factors for ovarian response in a corifollitropin alfa/GnRH antagonist protocol for controlled ovarian stimulation in IVF/ICSI cycles

\author{
Sergio Oehninger ${ }^{{ }^{*}}$, Scott M. Nelson ${ }^{2}$, Pierre Verweij ${ }^{3}$ and Barbara J. Stegmann ${ }^{4}$
}

\begin{abstract}
Background: This secondary analysis aimed to identify predictors of low ( $<6$ oocytes retrieved) and high ovarian response (>18 oocytes retrieved) in IVF patients undergoing controlled ovarian stimulation with corifollitropin alfa in a gonadotropin-releasing hormone $(\mathrm{GnRH})$ antagonist protocol.

Methods: Statistical model building for high and low ovarian response was based on the $150 \mu \mathrm{g}$ corifollitropin alfa treatment group of the Pursue trial in infertile women aged $35-42$ years $(n=694)$.

Results: Multivariable logistic regression models were constructed in a stepwise fashion ( $P<0.05$ for entry). $14.1 \%$ of subjects were high ovarian responders and $23.2 \%$ were low ovarian responders. The regression model for high ovarian response included four independent predictors: higher anti-Müllerian hormone (AMH) and antral follicle count (AFC) increased the risk, and higher follicle-stimulating hormone (FSH) levels and advancing age decreased the risk of high ovarian response. The regression model for low ovarian response also included four independent predictors: advancing age increased the risk, and higher AMH, higher AFC and longer menstrual cycle length decreased the risk of low ovarian response.
\end{abstract}

Conclusions: AMH, AFC and age predicted both high and low ovarian responses, FSH predicted high ovarian response, and menstrual cycle length predicted low ovarian response in a corifollitropin alfa/GnRH antagonist protocol.

Trial registration number: NCT01144416, Protocol P06029

Keywords: Predictive modelling, Ovarian response, Corifollitropin alfa, GnRH antagonist

\section{Introduction}

In assisted reproductive technology, both very low and very high ovarian responses to ovarian stimulation have been associated with increased cancellation rates and compromised pregnancy and live birth rates $[1,2]$. A high ovarian response also increases the risk for development of ovarian hyperstimulation syndrome (OHSS) [3]. Early identification of potential low and high responders is relevant to enable individualization of the ovarian stimulation treatment regimen [4].

The majority of studies on predictors of ovarian response have analyzed patients treated with recombinant ( $r$ ) follicle-

\footnotetext{
* Correspondence: OehninSC@EVMS.edu

${ }^{1}$ The Jones Institute for Reproductive Medicine, Eastern Virginia Medical School, 601 Colley Avenue, Norfolk, VA 23507-2007, USA

Full list of author information is available at the end of the article
}

stimulating hormone (FSH) in long gonadotropin-releasing hormone $(\mathrm{GnRH})$ agonist protocols. Systematic reviews have identified anti-Müllerian hormone (AMH), antral follicle count (AFC) and basal FSH as predictors of low ovarian response and $\mathrm{AMH}$ and $\mathrm{AFC}$ as predictors of high ovarian response in these protocols $[5,6]$, although the independence of these markers has not always been tested. AFC, basal FSH, luteinizing hormone (LH) and AMH have been identified as common prognostic factors for low or high ovarian response in rFSH GnRH antagonist protocols [7, 8].

Corifollitropin alfa is a novel recombinant gonadotropin, a single dose of which is capable of initiating and sustaining multifollicular growth during the first 7 days of ovarian stimulation as a replacement for 7 daily injections with $\mathrm{rFSH}$. The treatment regimen retains the 
capacity for flexibility to individualize treatment after day 7 [9]. A retrospective cohort study in young women treated with corifollitropin alfa in a $\mathrm{GnRH}$ antagonist protocol has shown that AMH and AFC are the best predictors for low and excessive response [10].

More women are delaying pregnancy, resulting in an increased number of women over the age of 35 years seeking infertility care [11]. The Pursue trial showed that in women aged 35-42 years, a single injection of $150 \mu \mathrm{g}$ corifollitropin alfa was noninferior to daily injections of $300 \mathrm{IU} \mathrm{rFSH}$ for the first 7 days of ovarian stimulation prior to in vitro fertilization (IVF) or intracytoplasmic sperm injection (ICSI) in terms of the vital pregnancy rate and was equally well tolerated with a low incidence of OHSS [12].

The objective of the current study was to identify predictors of low and high ovarian response in IVF/ICSI patients aged 35 to 42 years undergoing ovarian stimulation with corifollitropin alfa in a $\mathrm{GnRH}$ antagonist protocol, using data from the corifollitropin alfa arm of the Pursue trial.

\section{Materials and methods}

This was a secondary analysis of data collected in the Pursue trial $(N=1390)$ (Trial registration number: NCT01144416; Protocol P06029), a double-blind, randomized controlled trial of corifollitropin alfa versus daily injections of $\mathrm{rFSH}$ [12]. The trial was conducted in accordance with principles of Good Clinical Practice and was approved by the appropriate institutional review boards and regulatory agencies (Chesapeake IRB, Columbia (http://www.chesapeakeirb.com/)). Written informed consent was provided by all subjects. Infertile women aged 35-42 years with a body weight of $\geq 50 \mathrm{~kg}$ and body mass index (BMI) $\geq 18$ and $\leq 32 \mathrm{~kg} / \mathrm{m}^{2}$ received either a single injection of $150 \mu \mathrm{g}$ corifollitropin alfa or daily injections of $300 \mathrm{IU} \mathrm{rFSH}$ for the first 7 days of stimulation, followed by $\leq 300 \mathrm{IU} / \mathrm{d} \mathrm{rFSH}$ starting on stimulation day 8 , if needed. GnRH antagonist treatment, $0.25 \mathrm{mg} / \mathrm{d}$ ganirelix, was started on day 5 until final oocyte maturation with $250 \mu \mathrm{g}$ recombinant human chorionic gonadotropin [12]. Patients were excluded if they had a history of, or current, polycystic ovary syndrome.

Validated immunoassays were performed at a central laboratory on frozen serum samples to assess $\mathrm{FSH}, \mathrm{LH}$, estradiol $\left(\mathrm{E}_{2}\right)$ and progesterone $(\mathrm{P})$ concentrations as previously described [13]. Assessment of $\mathrm{AMH}$ was carried out using the validated Active AMH Gen II ELISA pre-mix assay from Beckman Coulter, Inc. (Brea, California, USA).

Limits of high and low ovarian response used in the current analyses were set at $>18$ oocytes retrieved and $<6$ oocytes retrieved, respectively.

\section{Statistical methods}

Initially, separate logistic regression models were constructed for high ovarian response $(>18$ oocytes retrieved or cycle canceled by the investigator because of too high response) and low ovarian response $(<6$ oocytes retrieved or cycle canceled due to insufficient response). Age was included as the first variable in both models. Other candidate prognostic factors were age at menarche (years), average menstrual cycle length (days), duration of infertility (years), BMI $\left(\mathrm{kg} / \mathrm{m}^{2}\right), \mathrm{AFC}$ and serum levels of FSH (IU/L), LH (IU/L), $\mathrm{E}_{2}(\mathrm{pmol} / \mathrm{L}), \mathrm{P}(\mathrm{nmol} / \mathrm{L})$ and $\mathrm{AMH}(\mathrm{ng} / \mathrm{mL})$ on day 1 of stimulation. For each candidate predictor, the association with high and low ovarian response was assessed using a $\chi^{2}$ test (the score test in a logistic regression model including only that predictor).

Multivariate logistic regression models were constructed in a stepwise fashion $(P<0.05$ for entry and $P>0.05$ for removal). Ten subjects with missing values $(1.5 \%)$ were excluded from model building, but were included in the estimation of the final model with missing covariate values imputed. For both outcomes the receiver operating characteristic (ROC) curve was plotted and the area under the curve (AUC, c-statistic) was calculated. This was done for the final model as well as for the intermediate models. These values were denoted 'apparent' AUCs. Optimismcorrected values were calculated using leave-one-out cross-validation (where the regression coefficients were re-estimated with each subject left out and then combined with the subject's covariate values in order to mimic the prediction of the outcome for each subject). The 'optimal' point on the ROC curve providing the best trade-off between sensitivity and specificity and the associated 'optimal' probability cutoff were identified. Sensitivity, specificity, positive predictive value and negative predictive value at the optimal cutoff were calculated.

Additionally, a combined model was constructed to predict both high and low ovarian by including the predictors that appeared in both models for the separate endpoints. The impact of leaving out prognostic factors that appeared in only one of the models was investigated. In the combined model, the regression coefficient of a given factor was allowed to differ between the outcomes of high and low ovarian response (i.e., proportional odds was not assumed).

External model validation was not possible as Pursue was the only study with corifollitropin alfa for which AMH measurements were available. Instead, models were internally validated by bootstrapping [14]. A total of 500 samples from 686 were drawn with replacement from the data set analyzed and for each sample, a logistic regression model was fitted for high, and separately, for low ovarian response using the stepwise approach described above. Each model was validated using the 
subjects not included in the bootstrap sample (on average, $36.8 \%$ ) [15]. Validation focused on discrimination-the ability of the model to distinguish between subjects with and without the event of interest, and calibration-the correspondence between the predicted event probabilities and observed proportions. Discrimination was measured by the AUC (or c-statistic) and calibration was measured by the calibration slope. Both quantities were obtained by fitting a logistic regression model in the validation sample with a single covariate for the so-called linear predictor, a combination of covariate values (from subjects in the validation sample) and regression coefficients (from the model constructed in the bootstrap sample). The calibration slope is the regression coefficient of the linear predictor, which should be close to unity. If the calibration slope is markedly less than one, this suggests that predictions should be 'shrunken' toward the mean when applied to future patients. The distribution of AUCs and calibration slopes was summarized over the 500 validation samples.

Finally, we compared our models with those developed by Polyzos et al. for excessive ( $>20$ oocytes retrieved) and poor ovarian response ( $<3$ oocytes retrieved). It should be noted, however, that the Polyzos et al. models were based on younger women from a single European center [10].

All analyses were performed using SAS PC version 9.3 (SAS Institute Inc., Cary, NC, USA).

\section{Results}

In this study population, $14.1 \%$ of women were high ovarian responders ( $>18$ oocytes retrieved) and $23.2 \%$ were low ovarian responders ( $<6$ oocytes retrieved). Descriptive statistics of potential predictors for ovarian response are shown in Table 1. These analyses showed that age at baseline, menstrual cycle length, AFC, FSH and AMH had a strong $(P<0.001)$ association with both high and low ovarian response.

\section{High ovarian response}

The logistic regression model for high ovarian response included four independent predictors (Table 2). Higher $\mathrm{AMH}$ concentrations and AFCs increased the risk for high ovarian response and higher FSH levels and advancing age decreased the risk. The apparent AUC of the ROC curve for the complete model predicting high ovarian response was 0.888 (Fig. 1). Correcting for the optimism associated with measuring the performance of the model in the same data set in which the model was constructed, the AUC was 0.880 (Table 2).

Table 2 and Fig. 1 also show that high ovarian response cannot be predicted by age alone (apparent AUC $=0.613$ ). Adding AMH strongly increased the ability of the model to separate patients with high ovarian response from those without high ovarian response (AUC $=0.864)$. Further inclusion of AFC and FSH also increased the performance of the model, but to a lesser extent (AUC $=0.888$ ). The sensitivity and specificity of the final model were $84 \%$ and $80 \%$, respectively (Table 3 ). The regression equation for the final model is given in Table 3 (first row). The equation can be used to calculate the probability for high ovarian response for any patient, given her age, $\mathrm{AMH}, \mathrm{AFC}$ and FSH. For a 38-year-old patient with $\mathrm{AMH}=1.8 \mathrm{ng} / \mathrm{mL}, \mathrm{AFC}=11$ and $\mathrm{FSH}=7.5 \mathrm{IU} / \mathrm{L}$, the

Table 1 Descriptive statistics of potential predictors (covariates) for ovarian response and their univariate correlation with high and low ovarian response

\begin{tabular}{|c|c|c|c|c|c|c|}
\hline Covariate & $\begin{array}{l}\text { Overall } \\
(n=686)\end{array}$ & $\begin{array}{l}\text { Low } \\
\text { (<6 oocytes }) \\
(n=159)\end{array}$ & $\begin{array}{l}\text { Normal } \\
(6-18 \text { oocytes }) \\
(n=430)\end{array}$ & $\begin{array}{l}\text { High } \\
(>18 \text { oocytes }) \\
(n=97)\end{array}$ & $\begin{array}{l}\text { High versus } \\
\text { normal/low } \\
(P \text {-value) }\end{array}$ & $\begin{array}{l}\text { Low versus } \\
\text { normal/high } \\
\text { (P-value) }\end{array}$ \\
\hline Age at baseline, $y$, mean (SD) & $38.0(2.2)$ & $38.6(2.2)$ & $37.9(2.1)$ & $37.3(2.1)$ & $<0.001$ & $<0.001$ \\
\hline Age at menarche, y, mean (SD) & $12.9(1.5)$ & $12.6(1.4)$ & $12.9(1.5)$ & $13.1(1.6)$ & 0.097 & 0.026 \\
\hline Average menstrual cycle length, days, mean (SD) & $28.2(1.7)$ & $27.5(1.5)$ & $28.3(1.7)$ & $28.7(1.7)$ & $<0.001$ & $<0.001$ \\
\hline Duration of infertility, $y$, mean (SD) & $2.8(2.8)$ & $2.5(2.3)$ & $2.9(2.8)$ & $3.2(3.2)$ & 0.169 & 0.100 \\
\hline $\mathrm{BMI}$ at baseline, $\mathrm{kg} / \mathrm{m}^{2}$, mean (SD) & $25.1(3.6)$ & $25.1(3.6)$ & $25.1(3.7)$ & $25.0(3.4)$ & 0.705 & 0.893 \\
\hline \multirow[t]{2}{*}{ AFC at day 1 of stimulation, n, mean (SD) } & $10.8(4.0)$ & $7.8(3.1)$ & $11.2(3.6)$ & $14.5(3.4)$ & $<0.001$ & $<0.001$ \\
\hline & $(n=679)$ & $(n=157)$ & $(n=426)$ & $(n=96)$ & & \\
\hline FSH at day 1 of stimulation, IU/L, median & 6.9 & 8.2 & 6.9 & 6.1 & $<0.001$ & $<0.001$ \\
\hline LH at day 1 of stimulation, IU/L, median & 4.6 & 4.2 & 4.7 & 4.7 & 0.555 & 0.089 \\
\hline Estradiol at day 1 of stimulation, pmol/L, median & 140.6 & 140.2 & 141.5 & 138.4 & 0.163 & 0.981 \\
\hline Progesterone at day 1 of stimulation, nmol/L, median & 1.9 & 1.9 & 1.9 & 2.0 & 0.984 & 0.849 \\
\hline $\mathrm{AMH}$ at day 1 of stimulation, $\mathrm{ng} / \mathrm{mL}$, median & 1.5 & 0.5 & 1.6 & 3.2 & $<0.001$ & $<0.001$ \\
\hline
\end{tabular}

Note: 8 subjects who did not have oocyte retrieval (for reasons other than too low or too high ovarian response according to the investigator) were excluded from the analysis

$S D$ standard deviation, $B M I$ body mass index, $A F C$ antral follicle count, FSH follicle-stimulating hormone, $L H$ luteinizing hormone, $A M H$ anti-Müllerian hormone 
Table 2 Logistic regression model for high ovarian response (>18 oocytes)

\begin{tabular}{lllllll}
\hline Covariate & OR & $95 \%$ & $\mathrm{Cl}$ & $P$-value & $\mathrm{AUC}^{\mathrm{a}}$ & $\mathrm{AUC}^{\mathrm{b}}$ \\
\hline Age (years) & 0.88 & 0.78 & 1.00 & 0.0590 & 0.613 & 0.545 \\
AMH (ng/mL) & 1.93 & 1.58 & 2.36 & $<0.0001$ & 0.864 & 0.858 \\
AFC (count) & 1.20 & 1.11 & 1.29 & $<0.0001$ & 0.882 & 0.876 \\
FSH (IU/mL) & 0.78 & 0.65 & 0.93 & 0.0055 & 0.888 & 0.880 \\
\hline
\end{tabular}

All odds ratios (OR) are per unit increase

$\mathrm{Cl}$ confidence interval, $A U C$ area under the curve, $A M H$ anti-Müllerian hormone,

$A F C$ antral follicle count, $F S H$ follicle-stimulating hormone

${ }^{\text {a Apparent }}$

${ }^{\mathrm{b} O p t i m i s m-c o r r e c t e d}$

linear predictor $\mathrm{LP}=-2.676$ and the probability for high ovarian response is 0.064 , or $6.4 \%$. For another 38-year-old patient with $\mathrm{AMH}=0.8 \mathrm{ng} / \mathrm{mL}, \mathrm{AFC}=8$ and $\mathrm{FSH}=8.5 \mathrm{IU} / \mathrm{L}$, the $\mathrm{LP}=-4.136$ and the probability for high ovarian response is 0.016 , or $1.6 \%$.

\section{Low ovarian response}

The multivariable regression model for low ovarian response also included four independent predictors (Table 4). Advancing age increased the risk for low ovarian response and higher $\mathrm{AMH}$, higher $\mathrm{AFC}$ and longer menstrual cycle length decreased the risk. The apparent AUC of the ROC curve for the complete model predicting low ovarian response was 0.886 (Fig. 2). The optimism-corrected AUC was 0.877 (Table 4).

Table 4 and Fig. 2 again show that low ovarian response cannot be predicted by age alone (apparent AUC $=0.605$ ). Adding AMH strongly increased the discriminative ability of the model (AUC $=0.871$ ), whereas further inclusion of AFC and menstrual cycle length also increased the performance of the model (AUC $=0.886$ ). The sensitivity and specificity of the final model were $77 \%$ and $87 \%$, respectively (Table 3 ). The regression equation for the final model is given in Table 3 (second row). For a 38-year-old patient with $\mathrm{AMH}=1.8 \mathrm{ng} / \mathrm{mL}$, $\mathrm{AFC}=11$ and a menstrual cycle length of 28 days, the linear predictor LP $=-2.548$ and the probability for low ovarian response is 0.073 , or $7.3 \%$. For the 38 -year-old patient with $\mathrm{AMH}=0.8 \mathrm{ng} / \mathrm{L}, \mathrm{AFC}=8$ and $\mathrm{FSH}=8.5 \mathrm{IU} / \mathrm{L}$, the $\mathrm{LP}=-0.359$ and the probability for low ovarian response is 0.411 , or $41.1 \%$.

\section{Combined model}

The regression models for high and low ovarian response had three predictors in common: age, AMH and AFC. The added value of FSH in the model for high ovarian response, although statistically significant, was not overwhelming. The same is true for menstrual cycle length in the model for low ovarian response. Without these factors, the AUC would decrease by only 0.006 and 0.004 for high and low ovarian response, respectively. Although age could also be dropped from the model without losing much predictive power, this factor was kept in the model as it is readily available. It should be noted that predicting high and low ovarian response based on the combined regression model should be based on different regression equations (Table 3 , third and fourth rows). For the 38-year-old patient with $\mathrm{AMH}=1.8 \mathrm{ng} / \mathrm{mL}$ and $\mathrm{AFC}=11$, the linear predictors for high and low ovarian response are -2.504 and -2.550 , respectively and the associated probabilities are $7.6 \%$ and $7.2 \%$, respectively (the estimates based on previous models were $7.3 \%$ and $7.3 \%$, respectively). The remaining

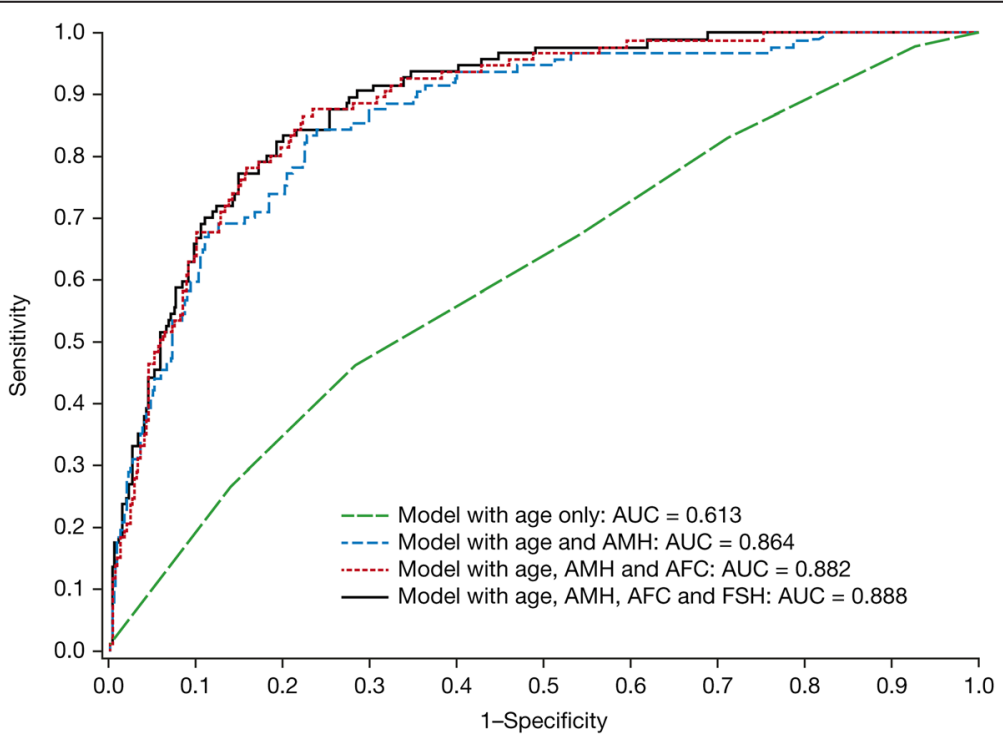

Fig. 1 Receiver operating characteristic curves for models for high ovarian response (>18 oocytes). AUC: area under the curve; AMH: anti-Müllerian hormone; AFC: antral follicle count; FSH: follicle-stimulating hormone 
Table 3 Test characteristics and equations for models for high and low ovarian response

\begin{tabular}{|c|c|c|c|c|c|}
\hline \multirow[b]{2}{*}{ Model } & \multirow[b]{2}{*}{ Cutoff } & \multirow[b]{2}{*}{ Sensitivity } & \multirow[b]{2}{*}{ Specificity } & \multicolumn{2}{|c|}{ Predictive value } \\
\hline & & & & Positive & Negative \\
\hline High ovarian response $^{a}$ & 0.15 & 0.84 & 0.80 & 0.41 & 0.97 \\
\hline Low ovarian response ${ }^{b}$ & 0.37 & 0.77 & 0.87 & 0.64 & 0.93 \\
\hline Combined high ovarian response ${ }^{c}$ & 0.13 & 0.87 & 0.78 & 0.39 & 0.97 \\
\hline Combined low ovarian response ${ }^{d}$ & 0.36 & 0.78 & 0.86 & 0.62 & 0.93 \\
\hline
\end{tabular}

Model-based probability $P=\frac{e^{L^{P}}}{1 e^{L P}}$ where $\mathrm{LP}$ is the linear predictor

${ }^{\mathrm{a}} \mathrm{LP}=0.6953-0.1232 \times$ age [years] $+0.6596 \times \mathrm{AMH}[\mathrm{ng} / \mathrm{mL}]+0.1829 \times \mathrm{AFC}$ [count] $-0.2517 \times \mathrm{FSH}[\mathrm{IU} / \mathrm{mL}]$

${ }^{b} \mathrm{LP}=5.1380+0.0961 \times$ age [years] $-1.6821 \times \mathrm{AMH}[\mathrm{ng} / \mathrm{mL}$ ] $-0.1690 \times \mathrm{AFC}$ [count] $-0.2304 \times \mathrm{CLn}$ [days]

${ }_{\mathrm{CP}}=-1.1213-0.1258 \times$ age [years] $+0.7010 \times \mathrm{AMH}[\mathrm{ng} / \mathrm{mL}]+0.1942 \times \mathrm{AFC}$ [count]

$\mathrm{d} \mathrm{LP}=-0.7701+0.0828 \times$ age [years] $-1.7373 \times \mathrm{AMH}[\mathrm{ng} / \mathrm{mL}]-0.1635 \times \mathrm{AFC}$ [count]

If the model-based predicted probability is above the cutoff, a patient would be classified as a potential high (respectively, low) responder

$A M H$ anti-Müllerian hormone, AFC antral follicle count, CLn cycle length, FSH follicle-stimulating hormone

$85.2 \%$ is the probability of a normal ovarian response between six and 18 oocytes.

Interpretation and application of the model would be simpler if age, AMH and AFC were classified as 'high' or 'low', for example, by using a threshold that optimizes sensitivity and specificity for each single factor. For high ovarian response, these thresholds are age $\leq 37$ years, $\mathrm{AMH} \geq 2.24 \mathrm{ng} / \mathrm{mL}$ and $\mathrm{AFC} \geq 13$ (details not shown). For low ovarian response, the values are age $\geq 39$ years, $\mathrm{AMH} \leq 1.03 \mathrm{ng} / \mathrm{mL}$ and $\mathrm{AFC} \leq 9$. However, it is well known that dichotomization of continuous covariates leads to loss of information. Indeed, the AUC of the simpler model for high ovarian response drops to 0.867 (from 0.882) and the AUC of the simpler model for high ovarian response drops to 0.841 (also from 0.882). For this reason, the simpler models were not pursued further.

\section{Model validation}

The bootstrap validation of the model for high ovarian response (including variable selection) resulted in a median AUC of 0.895 in the validation samples (2.5 and 97.5 percentage points: 0.862 and 0.923$)$. The median calibration slope was 0.990 (0.964-1.004). The validation of the model for low ovarian response based on the same 500 bootstrap samples resulted in a median AUC of $0.890(0.857-0.917)$ and a median calibration slope of

Table 4 Logistic regression model for low ovarian response (<6 oocytes)

\begin{tabular}{lcccccc}
\hline Covariate & OR & $95 \%$ & $\mathrm{Cl}$ & $P$-value & $\mathrm{AUC}^{\mathrm{a}}$ & $\mathrm{AUC}^{\mathrm{b}}$ \\
\hline Age (years) & 1.10 & 0.99 & 1.22 & 0.0711 & 0.605 & 0.553 \\
$\mathrm{AMH}(\mathrm{ng} / \mathrm{mL})$ & 0.19 & 0.12 & 0.28 & $<0.0001$ & 0.871 & 0.867 \\
AFC (count) & 0.85 & 0.78 & 0.91 & $<0.0001$ & 0.882 & 0.877 \\
Menstrual cycle length (days) & 0.79 & 0.69 & 0.92 & 0.0017 & 0.886 & 0.877 \\
\hline
\end{tabular}

Odds ratios are per unit increase

$\mathrm{Cl}$, confidence interval, $A U C$ area under the curve, $A M H$ anti-Müllerian hormone,

$A F C$ antral follicle count

${ }^{\text {a Apparent }}$

boptimism-corrected
0.937 (0.557-1.461). These results suggested good discrimination and calibration for high and low ovarian response.

\section{Comparison with the model of Polyzos et al.}

Our findings agree with those of Polyzos et al. [10] in that $\mathrm{AMH}$ and AFC are important predictors of ovarian response. Polyzos et al. developed models for excessive ovarian response ( $>20$ oocytes retrieved) and poor ovarian response ( $<3$ oocytes retrieved) based on $\mathrm{AMH}$ and AFC. The linear predictor for excessive ovarian response based on our data would be LP = $-7.287+0.664 \times \mathrm{AMH}+0.260 \times \mathrm{AFC}$ (details not shown), rather similar to the regression equation of Polyzos et al. $(z=-6.782+0.557 \times A M H+0.172 \times$ AFC $)$.

Our linear predictor for poor ovarian response, $\mathrm{LP}=0.964-2.710 \times \mathrm{AMH}-0.167 \times \mathrm{AFC}$, however, is different from their regression equation $(\mathrm{z}=2.161-0.991 \times \mathrm{AMH}-0.171 \times \mathrm{AFC})$. The main reason is that the percentage of poor responders in our data set is markedly lower than reported by Polyzos et al. (6.7 \% versus $34.3 \%)$. This difference could not be accounted for by possibly different values of $\mathrm{AMH}$ and $\mathrm{AFC}$ in our population. The percentages of excessive response were similar $(9.5 \%$ versus $8.6 \%$ ). Using the linear predictors of Polyzos et al. in our data resulted in calibration slopes of 1.31 and 1.72 for excessive and poor response, respectively. These were statistically significantly different from one $(P=0.032$ and $P=0.0032$, respectively), suggesting that these predictors should not be used for older women. Predictive models inevitably reflect the data set from which they are derived, which is why we made the validation effort.

\section{Discussion}

In the population of women in the Pursue trial aged 3542 years old undergoing ovarian stimulation with corifollitropin alfa in a $\mathrm{GnRH}$ antagonist protocol, the predictive factors for high ovarian stimulation were age, AFC, basal 


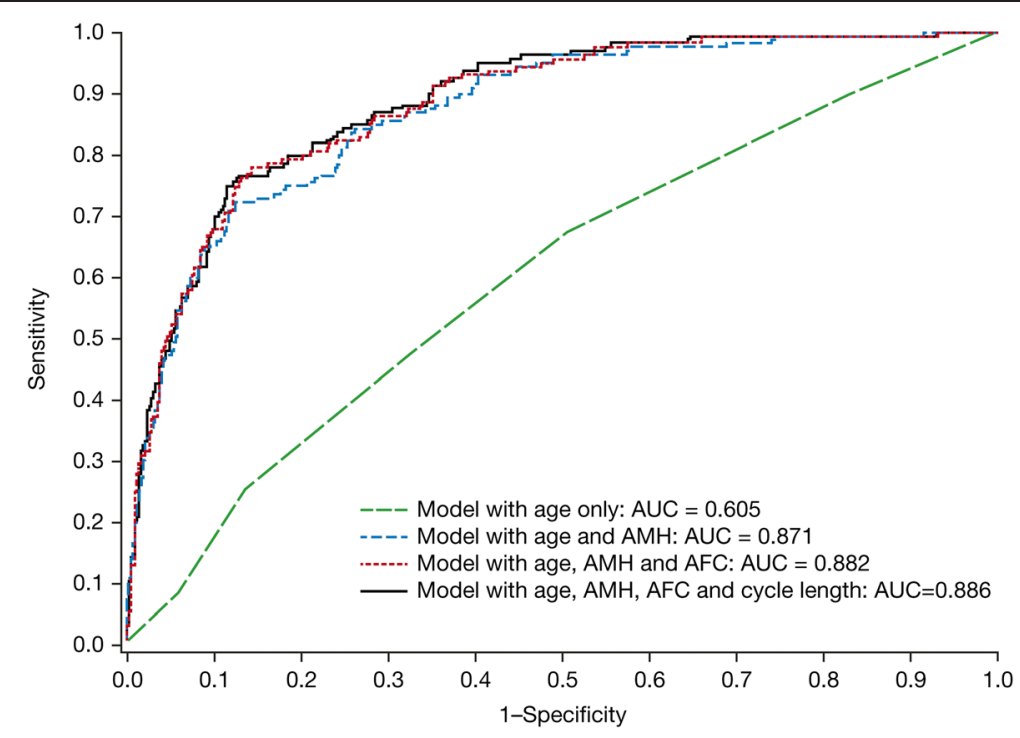

Fig. 2 Receiver operating characteristic curves for models for low ovarian response ( $<6$ oocytes). AUC: area under the curve; AMH: anti-Müllerian hormone; AFC: antral follicle count

FSH and $\mathrm{AMH}$, factors previously identified from $\mathrm{rFSH}$ GnRH agonist and antagonist protocols [5-8]. In the older patient population in the Pursue trial [12], increased menstrual cycle length was identified as a factor that decreased the risk of low ovarian response. Women with a history of polycystic ovary syndrome were excluded. Compared with younger women (18-36 years old) undergoing ovarian stimulation with $\mathrm{rFSH}$ in a $\mathrm{GnRH}$ antagonist protocol, in the current analyses, increased LH and BMI [16] were not identified as predictors of high ovarian response [8].

In this study, AMH concentrations were measured on frozen serum samples using the Active AMH Gen II ELISA pre-mix assay. For stored samples, there is no difference between the pre-mix and post-mix protocols in $\mathrm{AMH}$ concentrations, as complement degradation has already occurred resulting in minimal interference in the assay [17].

Limits of high ( $>18$ oocytes retrieved) and low ( $<6$ oocytes retrieved) ovarian response used in the current analyses were selected as subjects with more than 18 oocytes recovered have an increased risk of OHSS [3] and those with fewer than six oocytes recovered have a compromised chance of pregnancy $[1,2]$. These limits are consistent with previous publications on excessive and low ovarian response $[7,8]$.

In a retrospective analysis of a prospective randomized trial in which patients aged 18-36 years were treated with corifollitropin alfa or $\mathrm{rFSH}$ in a GnRH antagonist protocol, the ongoing pregnancy success rates in high responders (186 subjects with $>18$ oocytes) were at least as high as in patients with fewer than 18 oocytes [18]. This difference from the Sunkara analysis [1] may be related to the fact that these were women with a normal menstrual cycle and body weight range, and those with extremes in AFC and with polycystic ovary syndrome were excluded. Also, the Sunkara analysis was mainly an analysis of GnRH agonist cycles [1].

The current analyses of older women undergoing ovarian stimulation with corifollitropin alfa in a GnRH antagonist protocol uphold that age of the subject, AFC and $\mathrm{AMH}$ are strong predictors of ovarian response, as indicated in younger women undergoing ovarian stimulation with $\mathrm{rFSH}$ in a GnRH agonist or antagonist protocol.

We conclude that in women aged 35 to 42 years undergoing ovarian stimulation with corifollitropin alfa in a GnRH antagonist protocol, AMH, AFC and age at the start of stimulation were prognostic for both high and low ovarian response, in addition to FSH for high ovarian response and menstrual cycle length for low ovarian response.

\section{Abbreviations}

AFC: Antral follicle count; AMH: Anti-Müllerian hormone; AUC: Area under the curve; BMl: Body mass index; E2: Estradiol; FSH: Follicle-stimulating hormone; GnRH: Gonadotropin-releasing hormone; ICSI: Intracytoplasmic sperm injection; IVF: In vitro fertilization; LH: Luteinizing hormone; OHSS: Ovarian hyperstimulation syndrome; P: Progesterone; r: Recombinant; ROC: Receiver operating characteristic.

\section{Competing interests}

SO has received travel and honoraria fees from Merck \& Co., Inc. SMN has received consultancy fees from Merck \& Co., Inc., Merck Serono, Ferring Pharmaceuticals, Beckman Coulter and Roche Diagnostics. PV is a former employee of MSD BV, Oss. BJS is a current employee of Merck \& Co., Inc., Kenilworth, NJ and may own stock/stock options in the company.

\section{Authors' contributions}

SO and BJS took part in the conception and design of the study and acquisition of data. PV and BJS took part in analysis of the data and drafting the manuscript. All authors took part in the interpretation of data, critical 
review and revision of the manuscript, and in the final approval of the version to be published.

\section{Acknowledgements}

Editorial support was provided by Pam Milner of PAREXEL. This support was funded by Merck \& Co., Inc., Kenilworth, NJ. The authors also thank Christine McCrary Sisk and Kristen Lewis of Merck \& Co., Inc., Kenilworth, NJ, for editorial assistance. Presented at the European Society of Human Reproduction and Embryology $29^{\text {th }}$ Annual Meeting, London, United Kingdom; July 7-10, 2013.

\section{Funding}

Financial support for this study was provided by Merck \& Co., Inc., Kenilworth, NJ, USA.

\section{Author details}

${ }^{1}$ The Jones Institute for Reproductive Medicine, Eastern Virginia Medical School, 601 Colley Avenue, Norfolk, VA 23507-2007, USA. ${ }^{2}$ School of Medicine, University of Glasgow, Glasgow, UK. ${ }^{3}$ MSD BV, Oss, The Netherlands. ${ }^{4}$ Merck and Co., Inc., Kenilworth, NJ, USA.

Received: 17 April 2015 Accepted: 2 October 2015

Published online: 31 October 2015

\section{References}

1. Sunkara SK, Rittenberg V, Raine-Fenning N, Bhattacharya S, Zamora J, Coomarasamy A. Association between the number of eggs and live birth in IVF treatment: an analysis of 400135 treatment cycles. Hum Reprod. 2011;26:1768-74.

2. van der Gaast MH, Eijkemans MJ, van der Net JB, de Boer EJ, Burger CW, van Leeuwen FE, et al. Optimum number of oocytes for a successful first IVF treatment cycle. Reprod Biomed Online. 2006;13:476-80.

3. Papanikolaou EG, Pozzobon C, Kolibianakis EM, Camus M, Tournaye H, Fatemi HM, et al. Incidence and prediction of ovarian hyperstimulation syndrome in women undergoing gonadotropin-releasing hormone antagonist in vitro fertilization cycles. Fertil Steril. 2006;85:112-20.

4. Fauser BC, Diedrich K, Devroey P. Predictors of ovarian response: progress towards individualized treatment in ovulation induction and ovarian stimulation. Hum Reprod Update. 2008;14:1-14.

5. Broekmans FJ, Kwee J, Hendriks DJ, Mol BW, Lambalk CB. A systematic review of tests predicting ovarian reserve and IVF outcome. Hum Reprod Update. 2006;12:685-718.

6. Broer SL, Dolleman M, Opmeer BC, Fauser BC, Mol BW, Broekmans FJ. AMH and AFC as predictors of excessive response in controlled ovarian hyperstimulation: a meta-analysis. Hum Reprod Update. 2011;17:46-54.

7. Andersen AN, Witjes H, Gordon K, Mannaerts B. Predictive factors of ovarian response and clinical outcome after IVF/ICSI following a rFSH/GnRH antagonist protocol with or without oral contraceptive pre-treatment. Hum Reprod. 2011;26:3413-23.

8. Broekmans FJ, Verweij PJ, Eijkemans MJ, Mannaerts BM, Witjes H. Prognostic models for high and low ovarian responses in controlled ovarian stimulation using a GnRH antagonist protocol. Hum Reprod. 2014;29:1688-97.

9. Leader A, Devroey P, Witjes H, Gordon K. Corifollitropin alfa or rFSH treatment flexibility options for controlled ovarian stimulation: a post hoc analysis of the Engage trial. Reprod Biol Endocrinol. 2013;11:52.

10. Polyzos NP, Tournaye H, Guzman L, Camus M, Nelson SM. Predictors of ovarian response in women treated with corifollitropin alfa for in vitro fertilization/intracytoplasmic sperm injection. Fertil Steril. 2013;100:430-7.

11. Younis JS. Ovarian aging: latest thoughts on assessment and management. Curr Opin Obstet Gynecol. 2011;23:427-34.

12. Boostanfar R, Shapiro B, Levy M, Rosenwaks Z, Witjes H, Stegmann BJ, et al. A large, comparative, randomized double-blind trial confirming noninferiority of pregnancy rates for corifollitropin alfa compared to rFSH in a GnRH antagonist controlled ovarian stimulation protocol in older IVF patients. Fertil Steril. 2015;104:235-40.

13. Devroey P, Boostanfar R, Koper NP, Mannaerts BM, ljzerman-Boon PC, Fauser BC. A double-blind, non-inferiority RCT comparing corifollitropin alfa and recombinant FSH during the first seven days of ovarian stimulation using a GnRH antagonist protocol. Hum Reprod. 2009;24:3063-72.
14. Steyerberg EW, Harrell Jr FE, Borsboom GJ, Eijkemans MJ, Vergouwe Y, Habbema JD. Internal validation of predictive models: efficiency of some procedures for logistic regression analysis. J Clin Epidemiol. 2001;54:774-81.

15. Efron B, Tibshirani R. Improvements on cross-validation: the.632+ bootstrap method. J Am Stat Assoc. 1997;92:548-60.

16. Ledger WL, Fauser BC, Devroey P, Zandvliet AS, Mannaerts BM. Corifollitropin alfa doses based on body weight: clinical overview of drug exposure and ovarian response. Reprod Biomed Online. 2011;23:150-9.

17. Welsh P, Smith K, Nelson SM. A single-centre evaluation of two new anti-Mullerian hormone assays and comparison with the current clinical standard assay. Hum Reprod. 2014;29:1035-41.

18. Fatemi HM, Doody K, Griesinger G, Witjes H, Mannaerts B. High ovarian response does not jeopardize ongoing pregnancy rates and increases cumulative pregnancy rates in a GnRH-antagonist protocol. Hum Reprod. 2013;28:442-52.

\section{Submit your next manuscript to BioMed Central and take full advantage of:}

- Convenient online submission

- Thorough peer review

- No space constraints or color figure charges

- Immediate publication on acceptance

- Inclusion in PubMed, CAS, Scopus and Google Scholar

- Research which is freely available for redistribution 